\title{
System Size Dependence of Azimuthal Correlations in Relativistic Heavy Ion Collisions
}

\author{
Wolf G. Holzmann, for the PHENIX Collaboration \\ Department of Chemistry, SUNY Stony Brook, Stony Brook, NY 11794-3400, USA
}

\begin{abstract}
Systematic comparisons of jet pair correlations obtained in $\mathrm{Cu}+\mathrm{Cu}$ and $\mathrm{Au}+\mathrm{Au}$ collisions at $\sqrt{s_{N N}}=200 \mathrm{GeV}$ are presented. The measured jet-pair distributions for both systems show strong modification of the away-side jet. For the same number of participating nucleons, the modification does not show a strong dependence on the collision system. It is suggested that such comparisons can provide important constraints for models which predict specific path length dependent jet modification effects.
\end{abstract}

Keywords: system size, jet, correlations, heavy ion collisions, quenching, path length

PACS: 25.75.Dw

\section{INTRODUCTION}

Accumulating experimental evidence suggests that matter with energy density in excess of what is required for the creation of a deconfined phase of quarks and gluons (QGP), is produced in $\mathrm{Au}+\mathrm{Au}$ collisions at $\sqrt{s_{N N}}=200 \mathrm{GeV}$ at RHIC [1]. Azimuthal correlation measurements provide an important probe for the properties of this matter since they allow the study of (di)jets. Hard scattered partons traversing hot and dense QCD matter can lose energy before fragmenting into hadrons. Such an energy loss results in significant modification to jet properties in heavy ion collisions [2, 3, 4]. Indeed, the away-side jet in central and mid-central $\mathrm{Au}+\mathrm{Au}$ collisions at $\sqrt{s_{N N}}=200 \mathrm{GeV}$ has been found to be strongly modified both in yield and shape [5, 6, 7].

Jet modification is expected to be sensitive to several properties of the QCD medium, including the gluon density, formation time and the path length traversed by partons. Recently, much excitement has revolved around discussions of jet-induced mach shocks [9, 10], primarily because of a possible sensitivity to the viscosity and the speed of sound in the nuclear collision medium. A crucial outstanding issue of great importance is the detailed underlying mechanism for jet quenching. The resolution of this issue will undoubtedly rely on experimental constraints for the respective influence of energy density and path length on jet modification. Both vary with collision centrality.

Two particle correlation measurements relative to the reaction plane, have been used in an initial attempt to investigate path length effects [11]. They indicate sensitivity to the orientation of the reaction plane [11]. A complementary but simpler approach is to study jet-modification as a function of colliding system size.

It is well known [12] that the transverse energy per particle does not change significantly from SPS to RHIC energies $\left(\sqrt{s_{N N}} \approx 20-200 \mathrm{GeV}\right)$, suggesting that almost all of this beam energy increase goes into particle production. Recent preliminary results from the PHOBOS collaboration also indicate that the measured charged particle pseu- 
dorapidity density in $\mathrm{Cu}+\mathrm{Cu}$ and $\mathrm{Au}+\mathrm{Au}$ are essentially identical [within errors] for the same number of participating nucleons $\left(N_{\text {part }}\right)$. Taken together, these measurements suggest that a selection of the same $N_{\text {part }}$ in both collision systems targets matter with very similar energy density. On the other hand, the system sizes are different and should lead to different effective path lengths relevant to jet modification. Using a simple Glauber model and assuming an elliptic overlap region, one can estimate system size differences of the order of $20-30 \%$ for $N_{\text {part }} \approx 74$. If triggering on high $p_{T}$ particles primarily selects jets from the surface of the fireball [8], the differences in effective path lengths between both systems might be reduced.

\section{RESULTS AND SUMMARY}

The analysis presented here uses $\mathrm{Au}+\mathrm{Au}$ and $\mathrm{Cu}+\mathrm{Cu}$ data collected at the same collision energy $\left(\sqrt{s_{N N}}=200 \mathrm{GeV}\right)$ in the two central arms of the PHENIX detector. The detector setup is described elsewhere [13]. The data analysis is analogous to Ref. [7].

We define the correlation function as the ratio of two distributions in the azimuthal angle difference $(\Delta \phi)$ between particle pairs formed with one hadron from a high- $p_{T}$ "trigger" bin $\left(2.5<p_{T}<4.0 \mathrm{GeV} / \mathrm{c}\right)$ and another hadron from a lower "associated" $p_{T}$ selection $\left(1<p_{T}<2.5 \mathrm{GeV} / \mathrm{c}\right)$. A foreground distribution $N_{c o r}$ is constructed with correlated particle pairs from the same event, and a background distribution $N_{m i x}$ is obtained by randomly pairing particles from different events within the same multiplicity and vertex classes: $C(\Delta \phi) \propto N_{c o r}(\Delta \phi) / N_{\text {mix }}(\Delta \phi)$. Fig. 1(Left) shows correlation functions for several centrality selections in $\mathrm{Cu}+\mathrm{Cu}$ collisions, as indicated.
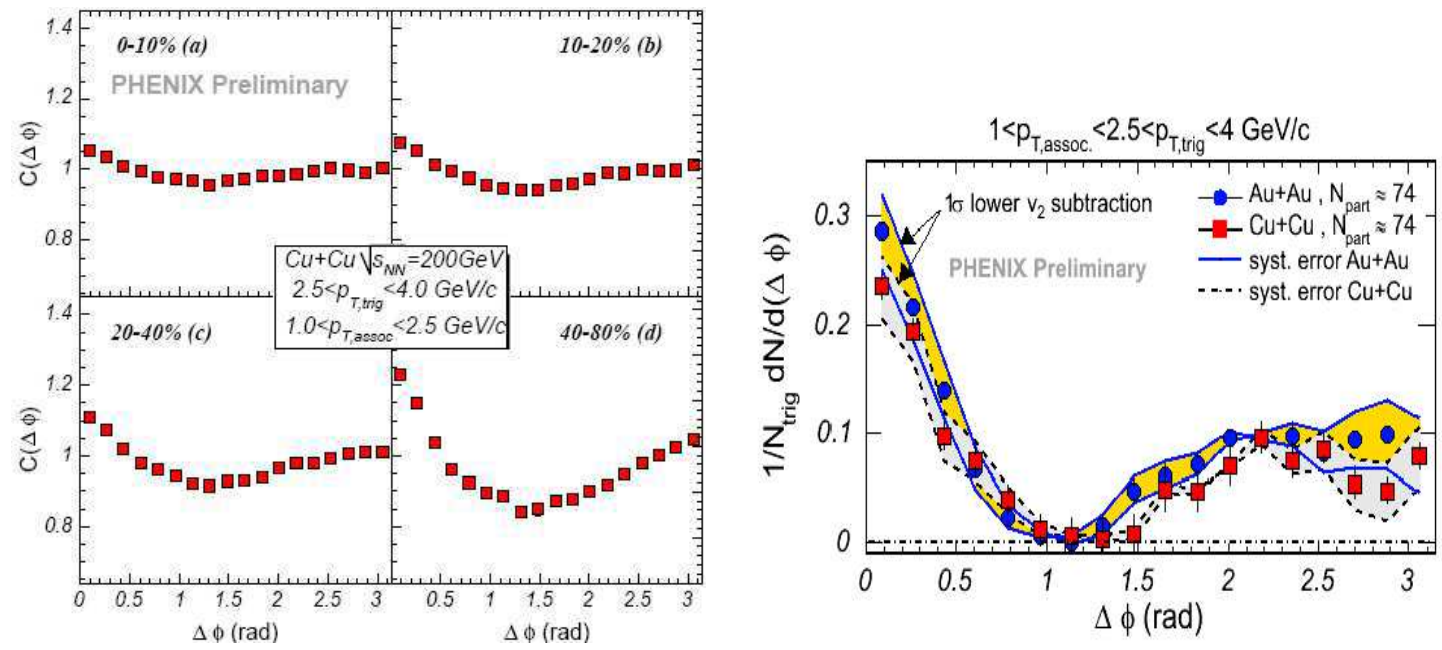

FIGURE 1. Left: Correlation functions for several centrality selections in $\mathrm{Cu}+\mathrm{Cu}$ as indicated. Right: Comparison of jet-pair distributions for $\mathrm{Au}+\mathrm{Au}$ (blue points) and $\mathrm{Cu}+\mathrm{Cu}$ (red squares) at $N_{\text {part }} \approx 74$.

For decomposition of the correlation functions, we assume that all correlation is only due to two sources: jet induced correlations and a harmonic component arising from elliptic flow. The harmonic amplitude is measured independently via a standard reaction plane analysis which minimizes non-flow correlations. This contribution is then subtracted from the correlation signal following the ZYAM constraint, detailed in 
Ref. [14]. This procedure gives the distribution of jet-correlated particle pairs per trigger particle.

Jet pair distributions for $\mathrm{Au}+\mathrm{Au}$ and $\mathrm{Cu}+\mathrm{Cu}$ collisions (for $N_{\text {part }} \approx 74$ ) are compared in Fig.1 (Right). The two distributions show clear indications for significant broadening of the away-side jet. However, a strong system size dependence is not evident, possibly suggesting that the energy density serves as the major actor in the jet modification process. The difference [albeit small] between the jet-pair distributions for the $\mathrm{Cu}+\mathrm{Cu}$ and the $\mathrm{Au}+\mathrm{Au}$ systems serves to constrain models which predict strong path-length effects in the hot and dense collision medium. A complimentary view is provided in Fig. 2 which compares the RMS widths extracted for the near- and away-side peaks for four separate centrality bins in $\mathrm{Cu}+\mathrm{Cu}$ with a recent $\mathrm{Au}+\mathrm{Au}$ analysis [7]. Although the away-side jet peaks are significantly broadened for mid-central and central $\mathrm{Cu}+\mathrm{Cu}$ and $\mathrm{Au}+\mathrm{Au}$ collisions, one can observe a smooth $N_{\text {part }}$ dependence of the away-side widths for both systems.

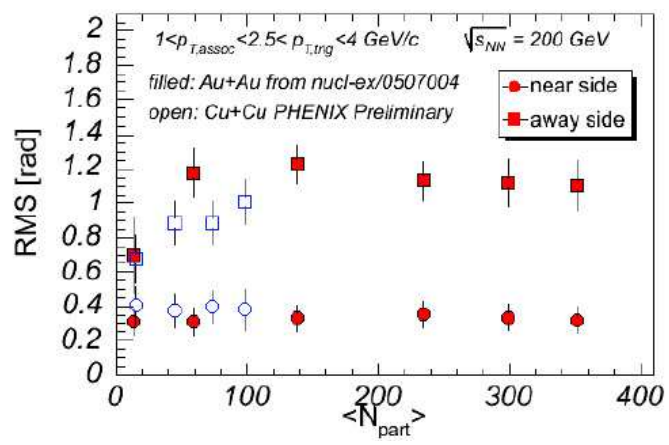

FIGURE 2. Comparison of RMS widths from near- and away-side peaks for four centrality bins in $\mathrm{Cu}+\mathrm{Cu}$ with the same quantities from a recent $\mathrm{Au}+\mathrm{Au}$ analysis [7]

In summary, we have presented a systematic comparison of the charged hadron azimuthal correlations between $\mathrm{Au}+\mathrm{Au}$ and $\mathrm{Cu}+\mathrm{Cu}$ collisions at $\sqrt{s_{N N}}=200 \mathrm{GeV}$. The jet pair distributions show significant broadening of the away-side jet in mid-central and central $\mathrm{Cu}+\mathrm{Cu}$ and $\mathrm{Au}+\mathrm{Au}$ collisions, but do not indicate a large system size dependence when the same $N_{\text {part }}$ and $p_{T}$ selections are made. This behavior suggests that the energy density serves as the major actor for jet modification. Further studies to quantitatively pin down the detailed path-length dependence of jet-modification are currently underway.

\section{REFERENCES}

1. K. Adcox et al., Nucl. Phys. A757 (2005) 184

2. J.D. Bjorken, (1982) FERMILAB-PUB-82-059-THY

3. D. A. Appel, Phys. Rev. D33, 717 (1986); J. P. Blaizot et al., Phys. Rev. D34, 2739 (1986)

4. X.N. Wang, M. Gyulassy, Phys. Rev. Lett. 68, 1480 (1992); X.N. Wang, Phys. Rev. C58, 2321 (1998)

5. C. Adler et al., Phys. Rev. Lett. 90082302 (2003)

6. J. Adams et al., Phys. Rev. Lett. 95152301 (2005)

7. S. S. Adler et al. [PHENIX Collaboration], nucl-ex/0507004

8. A. Dainese, C. Loizides and G. Paic hep-ph/0511045

9. H. Stöcker, Nucl. Phys. A750 (2005) 121; J. Casalderrey-Solana et al., (2004) hep-ph/0411315

10. J. Ruppert and B. Müller, Phys. Lett. B618 (2005) 123-130

11. J. Adams et al., Phys. Rev. Lett. 93252301 (2004) 
12. S. S. Adler et al., Phys. Rev. C71, 034908 (2005)

13. K. Adcox et al., Nucl. Instrum. Meth. A499 (2003) 469

14. N.N. Ajitanand et al., Phys. Rev. C72, 011902 (2005) 